\title{
A Method for Selecting the Next Best Angle-of-Approach for Touch-Based Identification of Beam Members in Truss Structures
}

\author{
Lili Bykerk, Phillip Quin, and Dikai Liu, Member, IEEE
}

\begin{abstract}
A robot designed to climb truss structures such as power transmission towers is expected to have adequate tactile sensing in the grippers to identify a structural beam member and its properties. Depending on how a gripper grasps a structural member, defined as the Angle-of-Approach (AoA), the extracted tactile data can result in erroneous identifications due to similarities in beam cross-sectional shapes and sizes. In these cases, further grasps at favourable Angles-of-Approach (AoAs) are required to correctly identify the beam member and its properties. This paper presents an information-based method which uses tactile data to determine the next best AoA for identification of beam members in truss structures. The method is used in conjunction with a state estimate of beam shape, dimension and AoA calculated by a Random Forest (RF) classifier. The method is verified through simulation by using data collected using a soft gripper retrofitted with simple tactile sensors. The results show that this method can correctly identify a structural beam member and its properties with a small number of grasps (typically fewer than 4). This method can be applied to other adaptive robotic gripper designs fitted with suitable tactile sensors, regardless of the number of sensors used and their layout.
\end{abstract}

Index Terms-Adaptive grasping, haptic glance, machine learning, object classification, object feature extraction, soft robotics, tactile sensors, transmission tower, truss structure.

\section{INTRODUCTION}

$\mathbf{T}$ RUSS structures, such as power transmission towers (Fig. 1a) are comprised mostly of extrusions of simple crosssectional shapes of varying dimension (Fig. 1b). A robot designed to climb a power transmission tower is expected to have appropriate tactile sensors in its grippers for safe and reliable climbing and operation. Tactile sensing in the grippers is crucial when considering the outdoor application environment, as the robot will encounter many imperfect lighting conditions for sensing with vision alone. In such scenarios, the sensory modality of touch can be used to identify structural beam members and their properties (such as cross-sectional shape, dimension and grasping Angle-of-Approach (AoA)) during climbing. Ideally, tactile data would be extracted by using the most efficient method which can seamlessly integrate into the climbing procedure. For this reason, a robotic "haptic glance" approach [1] would be more practical than other methods such as Exploratory Procedures (EPs) [2], [3].

This research is supported in part by the Centre for Autonomous Systems at the University of Technology Sydney, Australia and the Tokyo Electric Power Company, Japan.

All authors are with Centre for Autonomous Systems, University of Technology Sydney, 81 Broadway, Sydney, Australia (email: lili.bykerk@student.uts.edu.au,phillip.quin@uts.edu.au,dikai.liu@uts.edu.au)
Previous work in object identification using a soft gripper retrofitted with tactile sensors [4] showed that a single grasp, commonly referred to as a haptic glance extracted unique tactile data, or "tactile patterns" to identify a structural beam member and its properties. Classification accuracies of $~ 95 \%$ were directly related to the unique cross-sectional shapes of beam members in the object set, which produced unique tactile patterns during grasping. These results validated that simple tactile sensors, such as Force Sensitive Resistors (FSRs), are suitable sensors for this grasping application. However, in some cases, a beam member could not be identified with confidence using tactile data from a single haptic glance. Uncertainty in the grasping AoA, cross-sectional shape or dimension motivates the need for multiple strategic haptic glances to extract sufficient tactile data to correctly identify a beam member and its properties.

Naturally, the question that needs to be answered is how to select the next best AoA for grasping to rapidly identify the structural beam members. We answer this question by developing an information-based method for selecting the next best AoA (in a 2D plane) to identify a beam member and its properties using raw tactile sensor readings alone. Without the need for proprioception, this method can be applied to other adaptive gripper designs (such as soft grippers) fitted with suitable tactile sensors. As an example, this paper assumes that an array of individual FSRs are used, however many other analog tactile sensing options exist and could be used in place of FSRs. In the proposed method, a trained Random Forest (RF) classifier [5] provides an initial state estimate, i.e. perceived beam member cross-sectional shape, dimension and grasping AoA, after executing a single haptic glance and collecting tactile data. If the result of this classification is inconclusive, then the information-based method is used to select the next best AoA. This method consults a database of tactile data (obtained offline, also used to train the RF classifier). Based on the current predictions, candidate AoAs are generated and the next best AoA is determined as the candidate AoA where the predicted tactile patterns have the highest sum of variance (information score). Grasping at the calculated next best AoA is expected to provide sufficient data for unambiguous identification of the target beam member and its properties. The proposed method is verified by performing simulations with data collected using a soft gripper retrofitted with FSRs.

The remainder of this paper is organised as follows. Section II discusses the related state-of-the-art literature, with a focus 
towards touch based object identification methods by grasping with soft robotic grippers. Section III describes an informationbased method to identify objects with similar cross-sectional shape and dimension using tactile sensor data. Section IV describes the grasping mechanism and sensors used, target objects, data collection procedure and classifier training. Section $\mathrm{V}$ presents the simulation results and details the method's performance in two case studies. Finally, section VI concludes the paper and discusses future work.

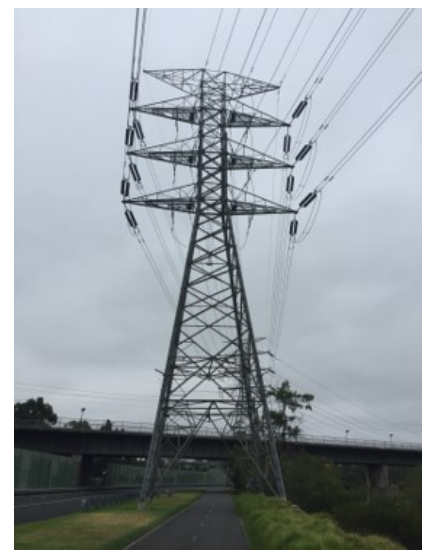

(a)

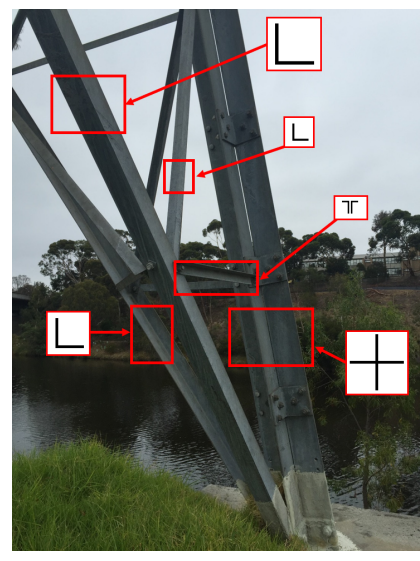

(b)
Fig. 1. (a) A power transmission tower in Melbourne, Australia, (b) Structural beam member in the tower with cross-sectional shapes and sizes shown.

\section{RELATED WORK}

Literature relating to object identification and recognition in robotics has predominantly focussed on vision sensing in controlled, indoor environments. In practical outdoor settings, however, many factors can reduce the reliability of vision sensing. It can therefore be advantageous to use touch as either a standalone or complementary sensory modality to vision. Robotic touch-based object recognition has naturally looked to mimic human EPs to extract as much information about a target object as possible [6]. A multitude of sensing configurations and hardware platforms can be combined with data extraction techniques to recognise and identify objects. Some methods use rigid robotic hands endowed with multiple sensory modalities and the ability to perform multiple EPs [7]-[14]. Whilst others focus on executing a single EP such as contour following [15]-[17], or repeatedly probing a target object to collect local "tactile images" [18]-[24]. Rigid grippers performing haptic glances for object recognition are of particular interest in this review. Early implementations used superquadric model-based methods to determine the shape of objects, using proprioceptive and tactile data [25], [26]. Recent developments have identified objects from a defined set using classifiers trained with data from grasping with anthropomorphic hands - using only joint sensor data [27] or both joint sensor and tactile data [28]-[30].

For brevity, the remainder of this literature review focuses on touch-based object identification and recognition methods using soft robotic grippers; specifically, by executing haptic glances. Methods and challenges for equipping soft robotic grippers with sensors are discussed, before object recognition techniques are analysed. Finally, strategies to perform multiple data collection actions for confident object identification in robotic grasping are compared and contrasted and the contribution of this research paper is summarised.

\section{A. Object Identification Using Soft Robotic Grippers}

Soft robotic grippers are continuum type mechanisms which inherently lack distinguishable and finite Degrees of Freedom (DoF). Since these mechanisms are typically designed to bend in plane during actuation, modelling their kinematics assumes either a Constant Curvature (CC) approximation [31] or a reduced kinematic Piecewise Constant Curvature (PCC) model [32]. Due to structural compliancy, knowledge of a gripper's specific configuration at any given time is difficult. To further complicate the problem, as a gripper interacts with the environment, unconstrained and unpredictable deformations can arise which limit the accuracy of these models. Sensor selection is therefore highly dependent on the gripper design, actuation method and grasping application. Recent literature in soft sensing has focussed on sensor design, with few integrations in grippers for object recognition purposes.

Methods of equipping soft robotic grippers with sensors have involved either retrofitting or embedding flexible or soft sensors within a gripper's structure during manufacturing a process which has become more viable as a result of additive manufacturing technologies. By assuming $\mathrm{CC}$, many different methods of proprioceptive sensing in soft robotics have emerged - where sensors typically change resistance with respect to an input force or strain. Some examples include the use of completely soft stretch and bend sensors embedded with liquid metals [33]-[35] or conductive elastomers [36][38]. Flexible bend sensors are also a popular off the shelf solution for proprioceptive sensing in soft robotics [39], [40], along with flexible force sensors for measuring point loads.

A number of factors prevent rigid robotic object recognition methods from being directly translated to the soft robotic domain. Uncertainty in kinematic models and interactions with target objects has generally resulted in the use of machine learning methods with sparse sensory inputs. Soft robotic object identification methods reported in recent literature have mostly used proprioceptive sensing only. For example, a soft pneumatic gripper with two embedded sensors - an air pressure sensor and a bend sensor - was able to recognise different sizes of spherical objects by monitoring the inflation pressure and bend sensor curvatures during pneumatic actuation [41]. A soft gripper with embedded resistive bend sensors [42] was designed to classify (using a k-Nearest Neighbours (k-NN) classifier) uniquely shaped and sized objects from a known set. During grasping, bend sensor readings were used to predict the hand configuration and classify the target object.

Few soft robotic grippers have incorporated both proprioceptive sensing and tactile sensing for object recognition. Some recent examples include a custom sensor skin for measuring deformation and contact, with the ability to construct 3D tactile object models [43]. Another design uses a four fingered gripper [44] with resistive bend sensors along the fingers and a force sensor in each of the fingertips for contact detection. The configuration of both hand and object can be 
detected and objects are identified using a k-NN classifier. A similar soft four fingered pneumatic gripper uses an embedded multi-layered arrangement of curvature and pressure sensors [45]. Using this system, objects are identified using a Support Vector Machine (SVM) classifier, although only trained with curvature sensor data. A related example uses an underactuated hybrid (rigid/soft) robot hand equipped with an array of barometric pressure sensors distributed along the grippers phalanges [46]. Object identification is performed with a RF classifier trained with grasping data from tactile sensors and actuator positions.

To the best of our knowledge, there has been a significant lack of soft or hybrid grippers possessing only an array of tactile sensors for object recognition. One identified example is a Fin Ray® style semi-rigid gripper with a retrofitted thin, flexible tactile sensor pad on the inner side of only one of the two fingers [47]. 50 tactile pressure images were collected during grasping of each of the 15 target objects (of unique shapes and sizes) and a Deep Convolutional Neural Network was trained.

\section{B. Selecting the Next Best Action for Confident Identification}

Depending on factors such as the gripper design, sensors, the types of target objects and how they are constrained in the application environment, the amount and quality of information obtained during a single haptic glance can vary. Often, several grasps are required to gain confidence in an objects' identity, pose and/or location in the environment.

Various methods exist for selecting the next best action for object identification or feature extraction using rigid robotic grippers. To identify an object from a set of household and industrial objects, a two fingered hand equipped with tactile sensors was used to collect low-resolution intensity images during grasping at various object heights [48]. Histogram intersection was used to find the next action (height at which to grasp the object) that would provide the highest expected information gain. An alternative method for object recognition uses a three-fingered robotic hand to actively explore objects [49]. Active behaviours are executed using a Bayesian approach to explore object locations that will reduce uncertainty.

A texture discrimination algorithm using a process called Bayesian exploration was used to determine a texture from a candidate set [50]. The algorithm adaptively selected the next optimal exploratory movement, based on previous experience by calculating the Bhattacharyya coefficient for two probability distribution functions. A decision to execute a movement is dependent on the potential information gained and if a higher level of confidence is worth the time and energy expenditure.

To localise an object in its environment, a tactile method for an autonomous robot was developed [51]. The next best touching action was determined using an information gain metric calculated by the Kullback-Leibler Divergence (KLD). Once the action which maximised the information gain was executed, the state of the object's pose was updated using an estimator (Bayes filter). The uncertainty was then checked against a threshold to determine if further actions were required for localisation.

\section{Research Contribution}

The discussed examples of robotic haptic perception have focussed on classification of a pre-defined set of (typically household) objects which vary greatly in their properties of size, stiffness and cross-sectional shape. These unique properties often result in rapid and reliable classifications using data collected from a single haptic glance. Few soft robotic grippers have been equipped with only tactile sensors for object recognition, as proprioception has been the major focus for soft robotic sensing. Existing methods combining proprioceptive and exteroceptive force sensing can be limited by a lack of informative data. For example, due to object size or grasping angles, a fingertip sensor might not always make contact with a target object [44]. In this paper, instead of relying on potentially erroneous proprioceptive data and a single fingertip sensor, the effectiveness of using an array of tactile sensors is investigated, and an information-based method is developed for confident object identification.

Our research focuses on identifying objects from a known object set which have similar (or even identical) crosssectional shape at certain Angles-of-Approach (AoAs) and identical stiffnesses. Whilst the problem is simplified by having a well defined object set for a particular type of truss structure (known a priori), the similarities of the different objects at particular AoAs increases the difficulty of the problem and leads to confusion in object identification. There are many scenarios where a single haptic glance is insufficient for confident object identification. Thoroughly grasping the object at differing AoAs for data collection is both time consuming and impractical. Ideally, a simple strategy to minimise the number of haptic glances and maximise the amount of information extracted would be developed. The general approach to the problem of where to touch next in object identification and localisation has relied on selecting an action to maximise the expected information gain (calculated probabilistically by KLD, Bhattacharyya distance or an alternative histogram comparison method) from a set of viable candidate actions. By maximising the information gained from each touch or grasp, a minimum number of touches is inherently achieved.

Our contribution in this work verifies the suitability of an array of simple tactile sensors for identifying objects with similar cross-sectional shape and dimension. In this paper, a simple method is presented for soft or other adaptive grippers which addresses the problem of selecting the next best grasping AoA for confident beam member identification. The next best AoA is selected based on the sum of the variance of collected tactile sensor data for a known set of objects (structural beam members). This method is generic in that it is not limited to a specific case of robotic manipulation and can therefore be used for other adaptive robotic grippers fitted with suitable tactile sensors.

\section{INFORMATION-BASED METHOD}

This section details an information-based method which can be implemented to identify objects with similar crosssectional shape and dimension by executing the minimum number of required grasps (haptic glances). In the context of 


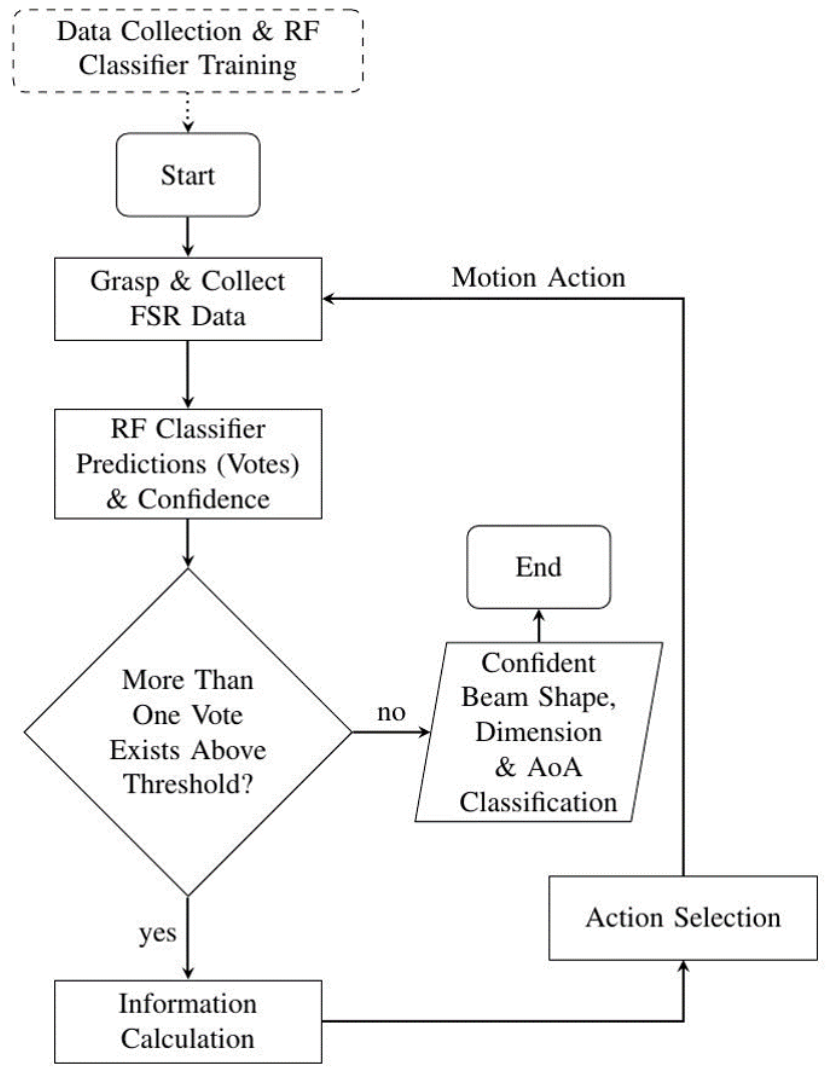

Fig. 2. Flow chart of beam identification using multiple haptic glances (information-based method).

the application environment (e.g. power transmission towers) there are two main scenarios where ambiguity arises in the classification of a structural beam member from a single haptic glance:

1) Multiple beam members can have similar cross-sectional shapes at certain AoAs, making it difficult to determine the true beam member shape e.g. disambiguating between a "T" beam and an "I" beam, and

2) Certain AoAs to a single beam member may also be similar, making it difficult to determine the true AoA of the gripper to the beam member e.g. due to symmetry.

Therefore, there are two key components to correctly identifying a beam member: determining the beam cross-sectional shape and the grippers' AoA to it. To narrow the field of potential beam member AoAs, haptic glances are performed at favourable AoAs, as determined by the information calculation process. The state space of the problem consists of all possible beam members $(\mathrm{Bm})$ and AoAs $(\mathrm{Na})$, or $\mathrm{Bm} \times \mathrm{Na}$ beam-angle pairs. The most informative AoA is the one which has the highest probability of eliminating the most beam-angle pairs from the set of candidates.

The method proposed in this section is illustrated in Fig. 2 and assumes that the robotic gripper used is equipped with appropriate tactile sensors, such as FSRs. An initial grasp provides FSR data which is used to gain predictions about the beam member using the RF classifier. Votes cast by the $\mathrm{RF}$ classifier are sorted in descending order for analysis. If a single vote exists above a user defined threshold, $\tau$, then this vote dominates the classification, the RF classifier is confident and only one haptic glance is required to identify the beam member and its properties.

If multiple votes exist above the threshold, then there may be confusion in the classification. The only case where multiple votes may exist above the threshold and result in confident classification is when the beam member being grasped is symmetrical. Symmetrical beam members contain repeated AoAs, i.e. where the data obtained from a given AoA is identical to another AoA shifted by a number of degrees, depending on the axis or axes of symmetry. If all votes cast above the threshold are for repeated AoAs to a symmetrical beam member, then no further grasping actions could be undertaken to disambiguate the AoAs. If there is confusion in the classification, then additional haptic glances are required to extract further data for confident identification of the structural beam member. At this step in the process, information for candidate AoAs is calculated.

Information is calculated by comparing the expected tactile patterns across $N a-1$ candidate AoAs (i.e. excluding the current perceived AoA). The expected tactile patterns for candidate AoAs are taken as the average of data from the number of grasps $(\mathrm{Ng})$ in the data collection phase - conducted offline. The candidate AoA, i.e. the AoA shift with the highest overall variance in the individual FSR analog readings (the next best AoA) is expected to provide the best possible data for unambiguous identification of the beam member and its properties. An action is selected based on the calculated next best AoA, and another grasp is performed. This process repeats until a single vote dominates the classification output, i.e. only one vote exists above the threshold and the beam member and AoA can be confidently identified.

Candidate AoAs for a grasping action are comprised of angle shifts in $\beta$ increments from the current predicted beam member AoAs. Therefore, to calculate information for candidate AoAs, the input matrix, $F$, of the FSR data differs, and is directly dependent on the initial votes cast by the RF classifier above the threshold.

Information is calculated (Equations 1, 2, 3) for the $\mathrm{Na}-1$ candidate AoAs (i.e. excluding the current perceived AoA, $\left.I=\left[I_{1}, I_{2}, \ldots, I_{N a-1}\right]\right)$. Information for a candidate AoA, $I_{a}$, is calculated by the sum of the variance $\left(\sigma^{2}\right)$ in the $N$ individual FSR readings for the $n$ predicted beam member AoAs.

$$
\begin{gathered}
\mu_{i}=\frac{1}{n} \sum_{j=1}^{n} F_{i j} \\
\sigma_{i}^{2}=\frac{1}{n} \sum_{j=1}^{n}\left(F_{i j}-\mu_{i}\right)^{2} \\
I_{a}=\sum_{i=1}^{N} \sigma_{i}^{2}
\end{gathered}
$$

The goal of the action selection is to choose the most favourable AoA for grasping, i.e. the AoA corresponding to $\max (I)$, which can provide sufficient data to unambiguously identify a beam member and its properties. 


\section{Experiment Setup And DATA Collection}

A data collection procedure was performed to extract FSR data during grasping of beam members from the object set listed in Table I. The data extracted was then used to train a RF classifier to be used in conjunction with the informationbased method described in Section III. This section further details the experimental setup, data collection procedure and classifier training.

TABLE I

Beam Members in the Target Object Set.

\begin{tabular}{ccc}
\hline $\begin{array}{c}\text { Cross-Sectional } \\
\text { Shape }\end{array}$ & $\begin{array}{c}\text { Cross-sectional } \\
\text { Dimensions (mm) }\end{array}$ & $\begin{array}{c}\text { AoAs for Data Collection } \\
\text { (in } \beta=10^{\circ} \text { increments) }\end{array}$ \\
\hline "L" & $50 \times 50$ & $0^{\circ} \rightarrow \pm 180^{\circ}$ \\
Square & $55 \times 75$ & $\pm 180^{\circ} \rightarrow-10^{\circ}$ \\
"+" & $50 \times 51$ & $0^{\circ} \rightarrow-80^{\circ}$ \\
"T" & $75 \times 75$ & $0^{\circ} \rightarrow-80^{\circ}$ \\
& $50 \times 50$ & $0^{\circ} \rightarrow \pm 180^{\circ}$ \\
"I" & $75 \times 75$ & $\pm 180^{\circ} \rightarrow-10^{\circ}$ \\
& $50 \times 50$ & $0^{\circ} \rightarrow-170^{\circ}$ \\
\hline
\end{tabular}

\section{A. Experiment Setup}

1) Experimental rig: The experimental rig (shown in Fig. 3) consisted of a soft gripper manufactured from Polyurethane elastomer F-180 A/B, Shore Durometer A $80 \pm 5$ (following the manufacturing process outlined in [52]). As described in [4], a customised linear actuator is used to generate grasping actions and an angle measurement tool is fixed to a beam member from the object set. The angle measurement tool was used to enable repeatable grasps at desired AoAs and distances from the surface of the beam members. The tool consisted of a circular structure with alignment holes at $\beta=10^{\circ}$ increments, located at varying radii from the centre of the beam members. These holes were used to lock the linear actuator to the angle measurement tool at the desired AoA for data collection. Since the information-based method only requires tactile sensor data, many different types of tactile sensors, including FSRs, could be used for data collection. In this research, the grasping surfaces of the gripper's fingers were retrofitted with Interlink 400 Short FSRs - three on each of the distal phalanges and six on the proximal phalanges, backed with 3D printed support pieces as shown in Fig. 3.

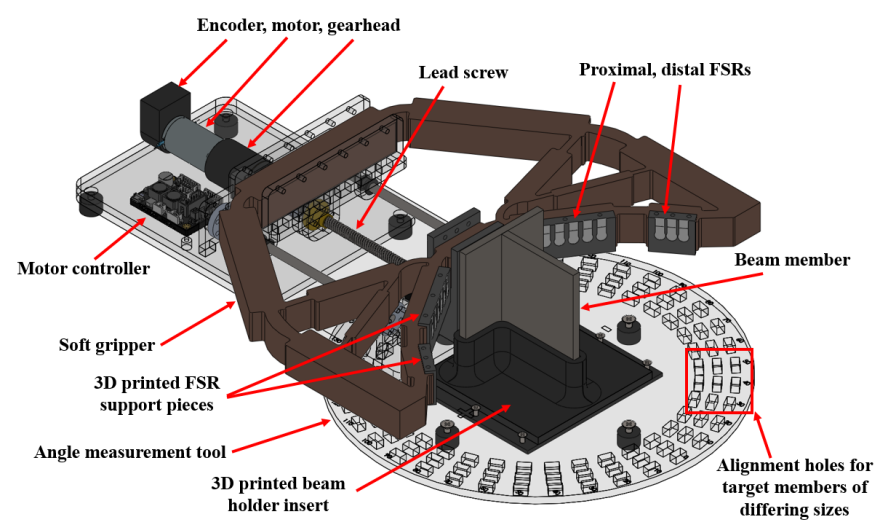

Fig. 3. CAD model - experimental rig setup.
2) Target objects: Target objects were chosen to represent a sample of possible power transmission tower structural beam members. Depending on the symmetry of the beam members, different AoAs were used for data collection in $\beta=10^{\circ}$ increments. For symmetrical members, fewer AoAs were used for data collection, due to repetition. The collected dataset is comprised of the $\mathrm{Bm}=9$ beam members with AoAs defined in Table I, totalling 207 unique beam-angle pairs. Fig. 4 shows the beam member cross-sectional shapes and their AoAs, defined as rotations about the $\mathrm{z}$-axis of the structural beam member. The complete dataset (populating the repeated AoAs for symmetrical beam members) is comprised of a total of $\mathrm{Bm}=9$ beams with $\mathrm{Na}=36\left(360^{\circ} / \beta\right)$ AoAs, therefore 324 beam-angle pairs.

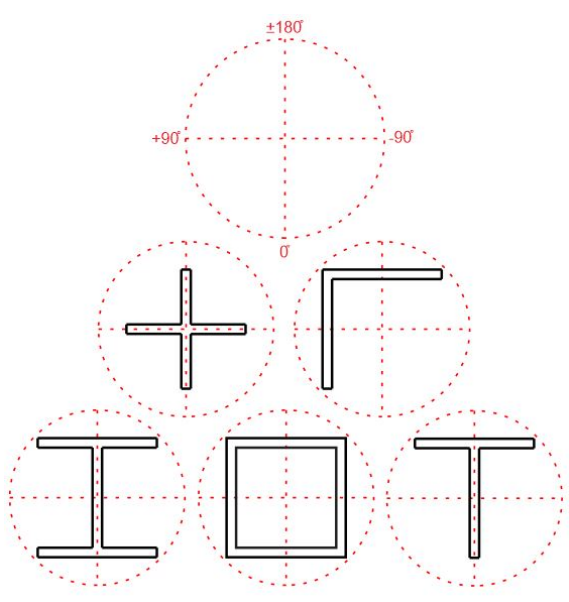

(a)

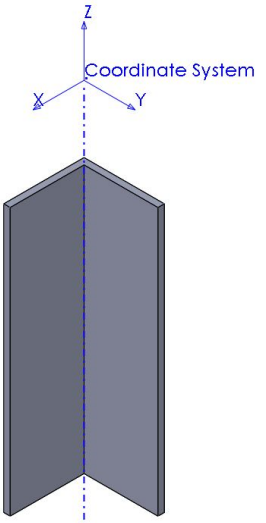

(b)
Fig. 4. (a) Possible beam member cross-sections and their AoA definitions, (b) Co-ordinate frame for orientations - only consider rotations about the zaxis i.e. gripper yaw.

\section{B. Data Collection}

The data collection procedure consisted of the following steps:

1) Attaching the angle measurement tool to a beam member using a 3D printed mounting piece,

2) Manually positioning the gripper to a desired AoA for data collection and locking the linear actuator base into the angle measurement tool,

3) Driving the linear actuator back and forth to open and close the gripper $N g=10$ times, recording FSR data for each haptic glance at the end of the actuator stroke length.

4) Manually shifting the gripper by $\beta=10^{\circ}$ and repeating the above steps for each of the AoAs listed in Table I.

For the purpose of consistency during data collection, the gripper was positioned to always approach beam members perpendicularly (i.e. as shown in Fig. 3) and at a set distance from the centre of the beam member. It should be noted that the gripper also sagged due to gravity, which was uncorrected during grasping.

The distance was selected by considering climbing in the practical application environment, where power grasps [53] are ideal. Executing power grasps typically results in strong 
and reliable grasps, with sufficient data for feature extraction since the gripper can make significant contact with the beam member surface/s. Increasing or decreasing the set distance would result in precision (pinch) grasps [53] at either the distal or proximal phalanges, respectively. Precision grasps are not desirable for the practical application environment as they are not sufficient for supporting the weight of a climbing robot. Additionally, precision grasps involve contacting an object at two points, which would not provide sufficient data for object identification. The general purpose of a grasp is to achieve maximum coverage of a beam member, resulting in the maximum number of sensors in contact. However, it is difficult to choose a distance that will result in power grasps being performed for all the beam members in the object set. When grasping the larger beam members, some precision grasps were executed, which caused ambiguity in the identification of the beam member (discussed further in Section $\mathrm{V}-\mathrm{C} 1$ ).

\section{Classifier Training}

Previous research [4] found that for a similar beam identification problem, a variety of common classifiers, including k-NN, Linear Discriminant Analysis (LDA), Multiclass SVM, Naïve Bayes, Bagged Trees Ensemble and RF were all suitable candidates resulting in high classification accuracies. In this previous work, from all of the classifiers trained and tested, a RF classifier with 100 trees provided one of the highest classification accuracies.

Any of these classifiers could be used for beam member identification using the information-based method presented in this paper. However, a RF classifier was chosen in this paper for the reason that they do not overfit (because of the Law of Large Numbers) and they are typically known as accurate classifiers due to the randomness [5]. In future work, the variable importance can also be examined to determine the effectiveness of the sensor placement on the gripper and the gripper design can be modified based on the results of this analysis.

Using the raw analog FSR data collected from the procedure described in Section IV-B, a RF classifier was trained with the goal of classifying the AoA, cross-sectional shape and dimension of beam members from within the defined target object set. The complete data set (containing data from the $N g=10$ repeated grasps at each of the 324 beam-angle pairs) was partitioned, with $90 \%$ of the data for training the classifier and $10 \%$ for testing the classifier. The 90:10 data split allowed for tactile patterns from each individual AoA to be used as haptic glance data.

\section{RESUlts AND Discussion}

\section{A. Beam Identification using a Single Haptic Glance}

Since the votes cast by the RF classifier dictate if the information-based method is required, the results of classification using the RF classifier alone are first analysed. This analysis reveals the misclassified AoAs (see Fig. 5), therefore providing insight into the AoAs which will provide interesting case studies using the information-based method.
1) Classification with the RF classifier only: High classification accuracies are expected when the objects being grasped significantly differ in dimension and/or cross-sectional shape. However, our dataset realistically represents the application environment, which consists of beam members with identical dimension and similar cross-sectional shape at certain AoAs.

The confusion matrix in Fig. 5 shows the RF classifier accuracy $(54.24 \%)$ for all of the possible $N a=36$ AoAs to each of the $B m=9$ beam members (324 beam-angle pairs) using a single haptic glance. It can be seen that confusion exists between multiple beam members, particularly those of identical dimension or between different AoAs to a single beam member.

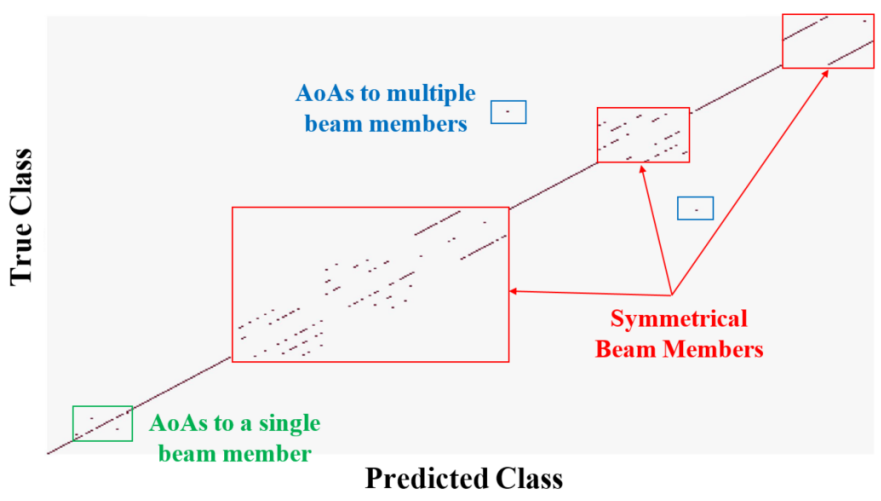

Fig. 5. Confusion matrix from the RF Classifier trained with 100 Trees on $90 \%$ of the complete dataset (training set). Results shown are the classifications of the remaining $10 \%$ of the data (test set). Total classification accuracy is $54.24 \%$.

Further analysis reveals that the confusion could be from either repeated AoAs (for symmetrical cross-sectional shapes, highlighted in red in Fig. 5), or from similar tactile patterns for given AoAs. The similarity in tactile patterns was an outcome of two possible grasping scenarios, where the gripper either:

- could not make adequate contact with the beam member during a grasp, highlighted in green in Fig. 5. Example data at these AoAs are shown in Fig. 6a, or

- was grasping a portion of a beam member with identical cross-sectional shape and dimension to another beam member, highlighted in blue in Fig. 5. Example data at these AoAs are shown in Fig. 6b, and Fig. 7 shows the gripper points of contact to the beam members which yield this data.

Upon removal of the repeated AoAs for the symmetrical beam members, the dataset could be reduced to 207 unique AoAs as listed in Table I. The classification accuracy with this reduced dataset was $88.4 \%$. For the 24 confused AoAs from the reduced dataset, both the beam member (crosssectional shape and dimension) and the grippers AoA cannot be confidently identified by using a single haptic glance.

Analysis of the RF classification results show that the classification output alone cannot be wholly trusted. By definition, the most frequent vote cast by each of the trees in the RF is the classification output. The classification result therefore simply needs to be higher in confidence than all other votes cast. In 


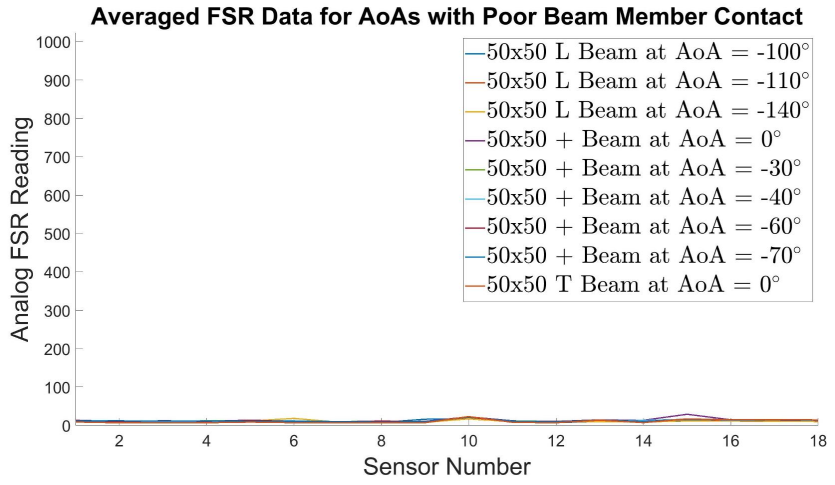

(a)

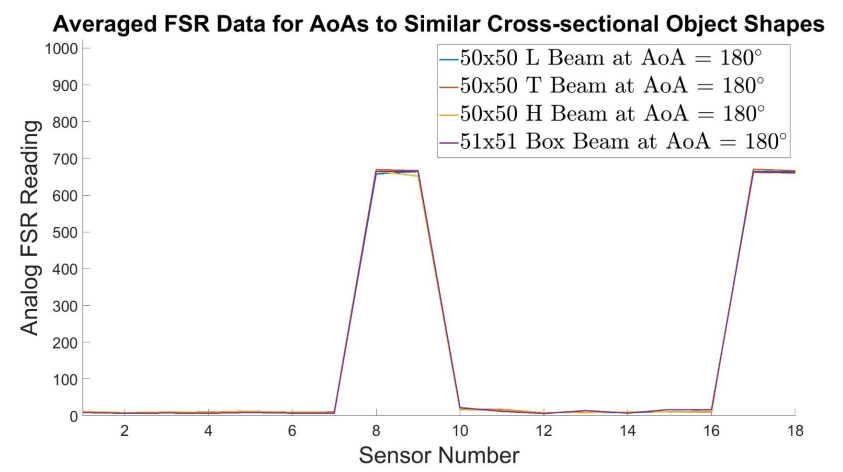

(b)

Fig. 6. Similar tactile patterns due to (a) Insufficient contact with the beam member during a grasp, (b) Grasping a portion of a beam member with identical cross-sectional shape to other beam members.

many cases, multiple AoAs may receive a significant portion of the votes, making the classification decision very close.

For these problematic "low information" AoAs, additional grasps would be required to sufficiently and confidently identify the beam member and its properties. This provides motivation for developing the information-based method.
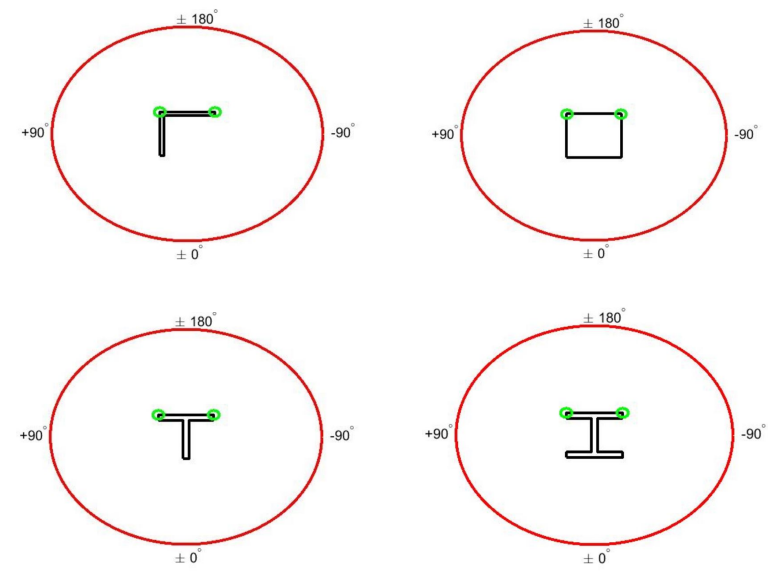

Fig. 7. Gripper points of contact when grasping beam members of similar cross-sectional shape at $180^{\circ}$.

2) Classification with the proposed information-based method: Similar to the RF classifier alone, using the information-based method still results in the majority of AoAs (82.4\%-96.6\%, depending on threshold value) being classified confidently with a single haptic glance (see Table II). In these cases, a single haptic glance provided unique tactile patterns for rapid and accurate identification of a beam member and its properties.

The RF classifier outputs the highest vote as the classification result, regardless of the number of votes cast and their confidence ratings. The information-based method, however, is used to analyse the individual RF classifier votes and assess whether or not the RF classifier output will be sufficient for unambiguous classification overall. This allows for any ambiguous AoAs to be flagged before they are classified (either correctly or incorrectly) and for further haptic glances to be executed as required. It is therefore possible that further ambiguous AoAs would arise from using the informationbased method, depending on the threshold value $(\tau)$ chosen.

An example of this can be observed by inputting FSR data from the AoA of $-120^{\circ}$ to the $50 \times 50$ " $\mathrm{T}$ " shaped beam member into the RF classifier. The three highest votes cast are: $\mathbf{3 1 \%}$ for $-120^{\circ} 50 \times 50$ "T", $\mathbf{3 0 \%}$ for $-30^{\circ} 50 \times 50$ "T" and $11 \%$ for $-120^{\circ} 50 \times 50$ "L". Whilst the RF classifier has correctly classified the AoA, it was very close to incorrectly classifying. According to the information-based method, this is an ambiguous AoA in the dataset. This AoA, however, is not considered ambiguous by the RF classifier alone, since it was correctly classified and would appear along the diagonal of the confusion matrix.

An example of misclassification by the RF classifier is the AoA of $-140^{\circ}$ to the $50 \times 50$ "L" shaped beam member. The three highest votes cast by the RF classifier are as follows: $\mathbf{2 1 \%}$ for $-110^{\circ} 50 \times 50$ "L", $\mathbf{1 9 \%}$ for $-140^{\circ} 50 \times 50$ "L" and $11 \%$ for $-100^{\circ} 50 \times 50$ "L".

For both of the AoA cases identified above, the informationbased method will flag these cases as ambiguous and decide to execute further strategic haptic glances to ensure that the beam member and its properties can be correctly identified. As shown by the results in Table II, in order to flag these ambiguous AoA cases, a threshold value must be selected appropriately.

\section{B. Beam Identification using Multiple Haptic Glances}

Table II summarises the results of the information-based method across all of the 324 AoAs in the dataset, with varying threshold values $(\tau)$. It can be clearly seen that the majority of beam member AoAs could be classified with a single haptic glance, regardless of the threshold value.

For the remaining AoAs where more than one haptic glance was required, typically the beam member and AoA could be identified with less than four haptic glances. The remainder of this section will further discuss the results obtained by choosing $\tau=15 \%$ which was shown to be the highest threshold that could achieve 100\% successful identification of the beam members and their properties.

When using the information-based method for beam member shape and AoA identification, the 41 (12.65\%) grasps which could not be identified using a single haptic glance could be classified confidently with more than 1 , but typically fewer than 4 haptic glances. One unique case existed where 5 haptic glances were required for beam member identification. 
TABLE II

RESULTS OF BEAM MEMBER IDENTIFICATION USING THE INFORMATION-BASED METHOD ACROSS THE 324 UNIQUE AOAS WITH VARYING THRESHOLD VALUES

\begin{tabular}{|c|c|c|c|c|c|c|c|}
\hline \multirow[t]{2}{*}{$\tau$} & \multicolumn{5}{|c|}{$\begin{array}{c}\text { Number of Haptic } \\
\text { Glances Required } \\
\text { For Correct } \\
\text { Classification }\end{array}$} & \multirow[t]{2}{*}{$\begin{array}{c}\text { Misclassified } \\
\text { AoAs }\end{array}$} & \multirow[t]{2}{*}{$\begin{array}{c}\text { AoAs } \\
\text { with All } \\
\text { Votes } \\
<\tau\end{array}$} \\
\hline & 1 & 2 & 3 & 4 & 5 & & \\
\hline $10 \%$ & 267 & 36 & 6 & 12 & 3 & 0 & 0 \\
\hline $15 \%$ & 283 & 30 & 4 & 6 & 1 & 0 & 0 \\
\hline $20 \%$ & 297 & 20 & 3 & 2 & 0 & 1 & 1 \\
\hline $25 \%$ & 305 & 14 & 1 & 0 & 0 & 1 & 3 \\
\hline $30 \%$ & 313 & 3 & 0 & 0 & 0 & 4 & 4 \\
\hline
\end{tabular}

Across the complete dataset, an average of 1.19 haptic glances were required for confident identification of the beam member and its properties.

The cases where more than two haptic glances were required for confident identification were examined carefully to determine if excessive grasping actions were executed as a result of the selection of the next best grasping AoA by the information-based method. In most of these cases, by greedily choosing the AoA with the highest calculated information, an ambiguous AoA was navigated to, therefore requiring additional haptic glance/s to confidently identify the beam member AoA.

\section{Case Studies}

The results are further examined in this section by analysing the two cases identified in Section V-A1 where a single haptic glance was not sufficient for the confident identification of the beam member and its properties. The first case study covers disambiguation between multiple beam member crosssectional shapes; where multiple AoAs to multiple beam members may receive votes above the threshold. The second case study covers disambiguation between multiple AoAs to a single beam member cross-sectional shape; where multiple AoAs to a single beam member may receive votes above the threshold.

1) Disambiguating between multiple beam member shapes: Disambiguating between multiple beam member shapes refers to the case identified by Fig. $6 \mathrm{~b}$ and Fig. 7, where votes may be cast by the RF classifier for multiple beam member crosssectional shapes and AoAs. The goal of the action selection is to choose the next best AoA that will narrow down the true beam member and AoA from the list of potential beams after any haptic glance.

When executing a primary haptic glance at $180^{\circ}$ to the $50 \times 50$ " $\mathrm{T}$ " beam member, the RF classifier casts votes (above the threshold) for many different beam member cross-sectional shapes ("T", "L", "I") and AoAs $\left(180^{\circ}, 0^{\circ}, 90^{\circ}\right)$. Upon initial confusion in the beam member cross-sectional shape from the first haptic glance, the information-based method calculates that the AoA located at an angle shift of $90^{\circ}$ Counterclockwise $(\mathrm{CCW})$ has the highest information. By navigating to this AoA, there is a high probability of eliminating the largest number of beam member cross-sectional shapes and AoAs from the list of possibilities. The information-based method also calculates that the AoA with the lowest information is located at a $10^{\circ}$ shift Clockwise $(\mathrm{CW})$.

As summarised by Fig. 8, by implementing the informationbased method to select the next best grasping AoA, the potential beam member cross-sectional shapes and AoAs are eliminated with one additional grasp. After the second haptic glance has been executed, the beam member and its properties are confidently identified and no further haptic glances are required for classification.

2) Disambiguating between multiple beam member AoAs: Disambiguating between multiple beam member AoAs refers to the case identified by Fig. 6a, where votes may be cast by the RF classifier for multiple AoAs to a single cross-sectional shape of beam member, e.g. $-100^{\circ},-110^{\circ},-140^{\circ}$ to the $50 \times 50$ " $L$ " shaped beam member. The goal of the action selection is to choose the next best AoA that will narrow down the true beam member AoA from the list of potential beam AoAs after any haptic glance.

Fig. 9a shows the votes cast by the RF classifier at the first grasping AoA of $-100^{\circ}$ to the $50 \times 50$ "L" shaped beam member. In this problem, the information-based method is seeking to disambiguate between the AoAs of $-100^{\circ}$ and $140^{\circ}$ after the first haptic glance. The remaining sub figures in column 1 of Fig. 9 show the votes cast by the RF classifier after performing haptic glances at the high information AoAs shown in column 2 of Fig. 9.

The high information cases for candidate grasps 3 and 4 show identical tactile patterns. After grasp 2 has been executed, the votes cast are separated by $90^{\circ}$. When rotating by $90^{\circ}$ to navigate to the high information AoA for grasp 3, the votes are still separated by $90^{\circ}$ and the information-based method is now seeking to disambiguate between $0^{\circ}$ and $90^{\circ}$. It should be noted that since only the $50 \times 50$ "L" beam is being considered for AoA disambiguation, that the vote cast for the $50 \times 50$ " $T$ " beam is discounted in grasp 3 . Finally, by rotating a further $90^{\circ}$ to reach the high information AoA shown by the tactile patterns in Fig. 9f, the RF classifier is once again seeking to disambiguate between $0^{\circ}$ and $90^{\circ}$, however with this additional rotation, now has absolute confidence in the identity of the beam member AoA. Thus, no further haptic glances are required to determine the beam member and its properties.

\section{Limitations}

As shown in Table II, the threshold value is a key limiting factor in the accuracy and efficiency of the information-based method. Setting this value too low can result in excessive grasping actions being required. Setting this value too high can result in erroneous classifications, or the information-based method failing to function at particular AoAs where all votes may be cast below the threshold value. Depending on the target object set and the tactile patterns obtained during grasping, an appropriate threshold value needs to be selected, such that its impact on the performance of the information-based method is optimal. This value can be selected by analysing the classification performance using the RF classifier only and observing the votes cast by the RF classifier for all misclassified AoAs. 


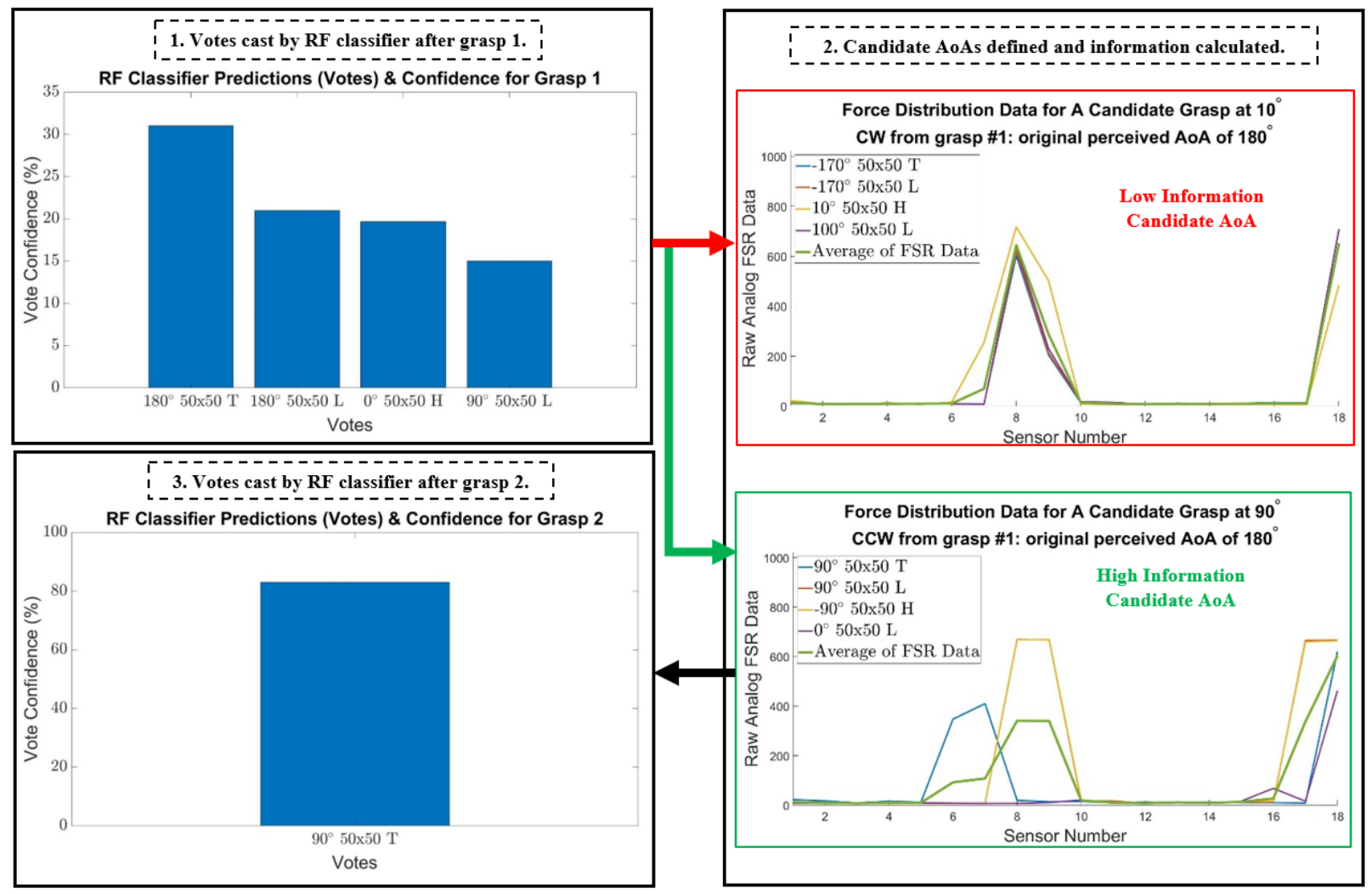

Fig. 8. Information-based method for grasping at the initial AoA of $180^{\circ}$ to the $50 \times 50$ " $\mathrm{T}$ " beam member.

\section{CONClusions AND Future Work}

In this paper, an information-based method was presented for selecting the next best grasping AoA, with the goal to achieve rapid and confident identification of a structural beam member and its properties by performing haptic glances. The method was tested and evaluated by using raw analog FSR data collected from grasping beam members of varying crosssectional shapes and dimensions with a soft robotic gripper. Using this method and the data from FSRs, it was found that for AoAs in the target object set, typically fewer than 4 haptic glances were required to correctly identify a structural beam member and its properties. Using the proposed method to execute multiple grasps, it was verified that an array of simple tactile sensors provided sufficient data for confident object identification. Furthermore, since this method only requires tactile sensing data, it can be used for other adaptive grippers with different types and arrangements of tactile sensors.

Future work includes further expanding the target object set to cover more structural beam members (i.e. to increase $\mathrm{Bm}$ ). The data collected could then be used to more rigorously test the information-based method in a practical setting and a threshold value $(\tau)$ could be appropriately selected for a more complete dataset. It is possible that with an expanded dataset, multiple votes may still exist above the threshold, but the beam member AoA can still be considered as confidently identified. In this case, an upper threshold value may also need to be defined to ensure that excessive grasping actions are not undertaken. Additionally, we envisage attaching the gripper to a robot arm and using the information-based method to identify the orientation of the structural beam member about the z-axis. Further investigation would also be conducted into determining the effects of varying the gripper AoA in the $\mathrm{x}$ and y axes (gripper roll and pitch).

\section{REFERENCES}

[1] R.L. Klatzky and S.J. Lederman, "Identifying objects from a haptic glance," Perception Psychophys., vol. 57, no. 8, pp. 1111-1123, 1995.

[2] S.J. Lederman and R.L. Klatzky, "Hand movements: A window into haptic object recognition," Cognitive Psychol., vol. 19, no. 3, pp. 342368, 1987.

[3] S.J. Lederman and R.L. Klatzky, "Extracting object properties through haptic exploration," Acta Psychologica, vol. 84, no. 1, pp. 29-40, 1993.

[4] L. Bykerk and D. Liu, "Experimental verification of a completely soft gripper for grasping and classifying beam members in truss structures," in Proc. IEEE/ASME Int. Conf. Adv. Intell. Mechatron., 2018, pp. 756761.

[5] L. Breiman, "Random Forests," Mach. Learning, vol. 45, no. 1, pp. 5-32, 2001.

[6] R. Klatzky, R. Bajcsy and S. Lederman, "Object exploration in one and two fingered robots," in Proc. IEEE Int. Conf. Robot. Autom., 1987, pp. 1806-1809.

[7] P. K. Allen, "Integrating Vision and Touch for Object Recognition Tasks," Int. J. Robot. Res., vol. 7, no. 6, pp. 15-33, 1988.

[8] A.M. Okamura, M.L. Turner and M.R. Cutkosky, "Haptic exploration of objects with rolling and sliding," in Proc. IEEE Int. Conf. Robot. Autom., 1997, pp. 2485-2490.

[9] J. Sinapov and A. Stoytchev, "Object category recognition by a humanoid robot using behavior-grounded relational learning," in Proc. IEEE Int. Conf. Robot. Autom., 2011, pp. 184-190.

[10] V. Chu, I. McMahon, L. Riano, C.G. McDonald, Q. He, J.M. PerezTejada, M. Arrigo, N. Fitter, J.C. Nappo, T. Darrell and K.J. Kuchenbecker, "Using robotic exploratory procedures to learn the meaning of haptic adjectives," in Proc. IEEE Int. Conf. Robot. Autom., 2013, pp. 3048-3055. 


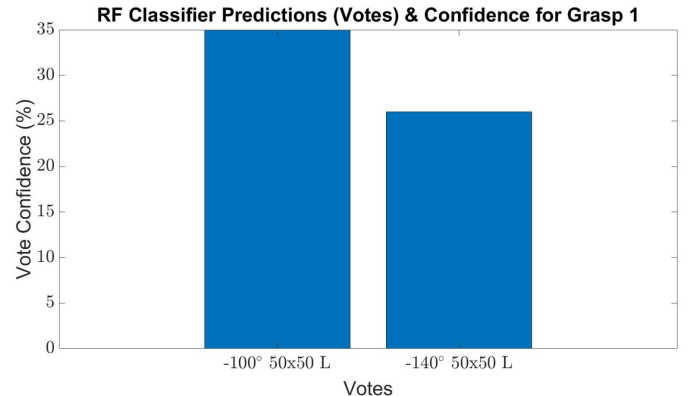

(a)

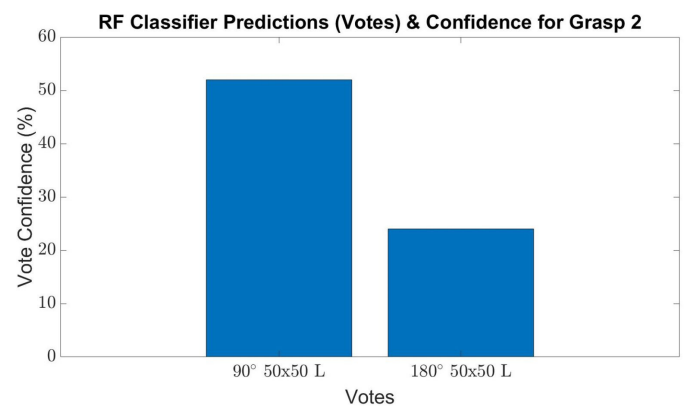

(c)

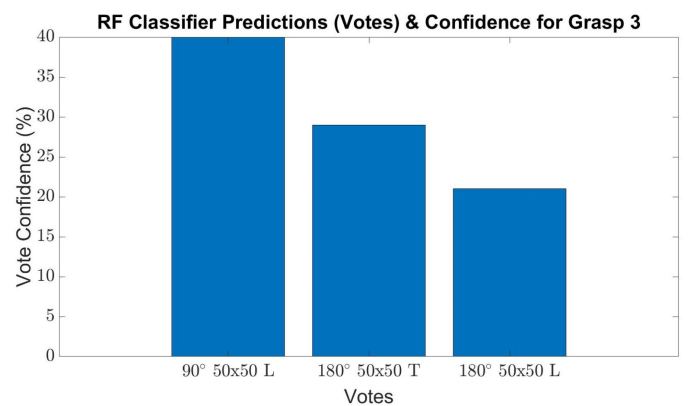

(e)

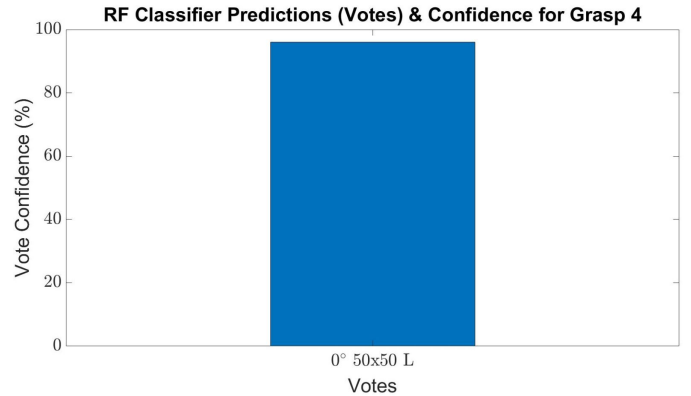

(g)

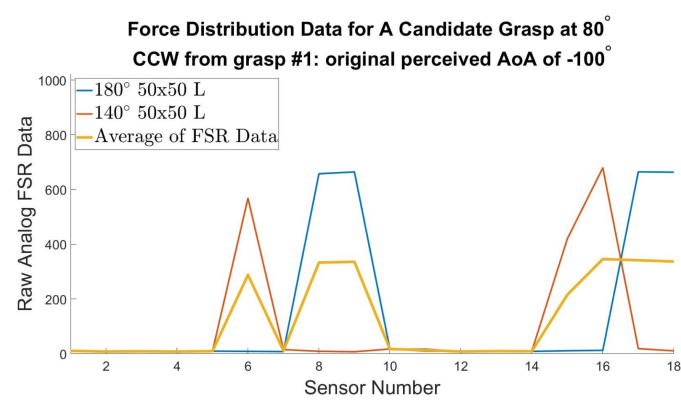

(b)

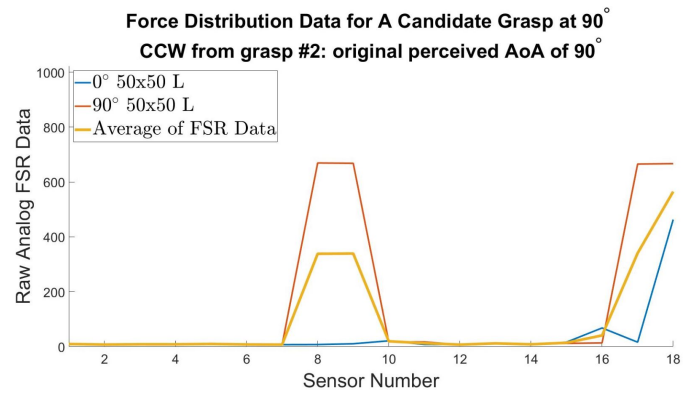

(d)

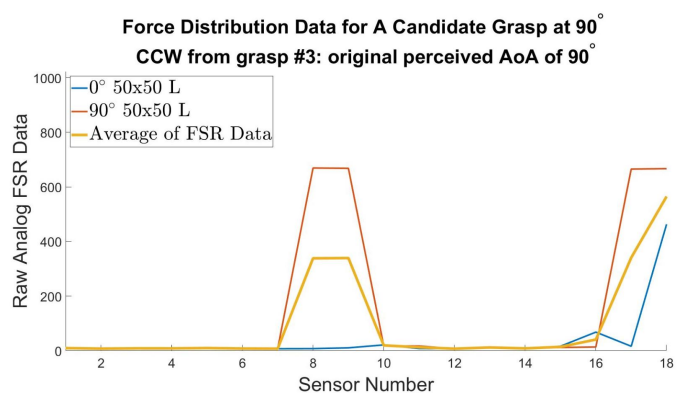

(f)

\section{No further grasping actions required. Beam member} and AoA confidently identified after 4 grasps.

Fig. 9. Column 1: RF classifier votes above threshold $(\tau=15 \%)$ for grasps $1-4$. (a): Original grasping AoA of $-100^{\circ}$ to the $50 \times 50$ "L" beam member. (c), (e), (g): High information grasping AoAs shown in column 2. Column 2: High information candidate AoAs, based on $\beta=10^{\circ}$ incremental angle shifts from the initial grasp data obtained at the AoA of $-100^{\circ}$ to the $50 \times 50$ "L" beam member. $(b),(d),(f)$ : High information candidate AoAs based on the classifications for grasps 1-3. $(\mathrm{g})$ : Beam member and AoA are correctly identified at this point, no further grasps required.

[11] M. Bjorkman, Y. Bekiroglu, V. Hogman and D. Kragic, "Enhancing visual perception of shape through tactile glances," in Proc. IEEE/RSJ Int. Conf. Intell. Robots Syst., 2013, pp. 3180-3186.

[12] R.D.P. Wong, R.B. Hellman and V.J. Santos, "Haptic exploration of fingertip-sized geometric features using a multimodal tactile sensor," in SPIE Sensing Technology and Applications, pp. 911605911605, International Society for Optics and Photonics, 2014.

[13] B. Higy, C. Ciliberto, L. Rosasco and L. Natale, "Combining sensory modalities and exploratory procedures to improve haptic object recognition in robotics," in Proc. IEEE-RAS 16th Int. Conf. on Humanoid Robots, 2016, pp. 117-124.

[14] N. Sommer and A. Billard, "Multi-contact haptic exploration and grasping with tactile sensors," Robot. Auton. Syst., vol. 85, pp. 48-61, 2016.

[15] A.M. Okamura and M.R. Cutkosky, "Feature Detection for Haptic Exploration with Robotic Fingers," Int. J. Robot. Res., vol. 20, no. 12, 
pp. 925-938, 2001.

[16] U. Martinez-Hernandez, T.J. Dodd, L. Natale, G. Metta, T.J. Prescott and N.F. Lepora, "Active contour following to explore object shape with robot touch," in Proc. World Haptics Conf., 2013, pp. 341-346.

[17] N.F. Lepora, K. Aquilina and L. Cramphorn, "Exploratory tactile servoing with active touch," IEEE Robot. Autom. Lett., vol. 2, no. 2, pp. 1156-1163, 2017

[18] R.A. Russell, "Object recognition by a 'smart' tactile sensor," in Proc. Australian Conf. Robot. Autom., 2000, pp. 93-98.

[19] G. Heidemann and M. Schopfer, "Dynamic tactile sensing for object identification," in Proc. IEEE Int. Conf. Robot. Autom., 2004, pp. 813818.

[20] Z. Pezzementi, E. Plaku, C. Reyda and G.D. Hager, "Tactile-Object Recognition From Appearance Information, IEEE Trans. Robot., vol. 27, no. 3, pp. 473-487, 2011.

[21] H. Liu, X. Song, T. Nanayakkara, L.D. Seneviratne, K. Althoefer, "A computationally fast algorithm for local contact shape and pose classification using a tactile array sensor," in Proc. IEEE Int. Conf. Robot. Autom., 2012, pp. 1410-1415.

[22] T. Corradi, P. Hall, P. Iravani, "Bayesian tactile object recognition: Learning and recognising objects using a new inexpensive tactile sensor," in Proc. IEEE Int. Conf. Robot. Autom., 2015, pp. 3909-3914.

[23] G. Vezzani, N. Jamali, U. Pattacini, G. Battistelli, L. Chisci and L. Natale, "A novel Bayesian filtering approach to tactile object recognition," in Proc. 16th IEEE-RAS Int. Conf. Humanoid Robot., 2016, pp. 256263.

[24] S. Luo, W. Mou, K. Althoefer and H. Liu, "Novel Tactile-SIFT Descriptor for Object Shape Recognition," IEEE Sens. J., vol 15, no. 9, pp. 5001-5009, 2015.

[25] P.K. Allen and K.S. Roberts, "Haptic object recognition using a multifingered dextrous hand," in Proc. IEEE Int. Conf. Robot. Autom., 1989, pp. 342-347.

[26] S. Caselli, C. Magnanini and F. Zanichelli, "Haptic object recognition with a dextrous hand based on volumetric shape representations," in Proc. Int. Conf. Multisensor Fusion and Integration for Intell. Syst., 1994, pp. 280-287.

[27] M. Johnsson and C. Balkenius, "Experiments with proprioception in a self-organizing system for haptic perception," in Proc. TAROS, 2007, pp. $239-245$.

[28] S. Takamuku, A. Fukuda and K. Hosoda, "Repetitive grasping with anthropomorphic skin-covered hand enables robust haptic recognition," in IEEE/RSJ Int. Conf. Intell. Robots Syst., 2008, pp. 3212-3217.

[29] N. Gorges, S. E. Navarro, D. Goger and H. Worn, "Haptic object recognition using passive joints and haptic key features," in Proc. IEEE Int. Conf. Robot. Autom., 2010, pp. 2349-2355.

[30] H. Soh and Y. Demiris, "Incrementally Learning Objects by Touch: Online Discriminative and Generative Models for Tactile-Based Recognition," IEEE Trans. Haptics, vol. 7, no. 4, pp. 512-525, 2014.

[31] M.W. Hannan and I.D. Walker, "Kinematics and the Implementation of an Elephant's Trunk Manipulator and Other Continuum Style Robots," J. Robot. Syst., vol. 20, no. 2, pp. 45-63, 2003.

[32] R.J. Webster and B.A. Jones, "Design and Kinematic Modeling of Constant Curvature Continuum Robots: A Review," Int. J. Robot. Res., vol. 29, no. 13, pp. 1661-1683, 2010.

[33] N. Farrow and N. Correll, "A soft pneumatic actuator that can sense grasp and touch," in Proc. IEEE/RSJ Int. Conf. Intell. Robots Syst., 2015, pp. 2317-2323.

[34] R.A. Bilodeau, E.L. White and R.K. Kramer, "Monolithic fabrication of sensors and actuators in a soft robotic gripper," in Proc. IEEE/RSJ Int. Conf. Intell. Robots Syst., 2015, pp. 2324-2329.

[35] J. Morrow, H. Shin, C. Phillips-Grafflin, S. Jang, J. Torrey, R. Larkins, S. Dang, Y. Park and D. Berenson, "Improving Soft Pneumatic Actuator fingers through integration of soft sensors, position and force control, and rigid fingernails," in Proc. IEEE Int. Conf. Robot. Autom., 2016, pp. 5024-5031.

[36] Y. Yang and Y. Chen, "Innovative Design of Embedded Pressure and Position Sensors for Soft Actuators," IEEE Robot. Autom. Lett., vol. 3, no. 2, pp. 656-663, 2018.

[37] R.L. Truby, M. Wehner, A.K. Grosskopf, D.M. Vogt, S.G.M. Uzel, R.J. Wood and J.A. Lewis, "Soft Somatosensitive Actuators via Embedded 3D Printing," Adv. Mat., vol. 30, no. 15, pp. 1706383, 2018.

[38] R.P. Rocha, P.A. Lopes, A.T de Almeida, M. Tavakoli and C. Majidi, "Fabrication and characterization of bending and pressure sensors for a soft prosthetic hand," J. Micromechanics and Microengineering, vol. 28, no. 3, pp. 034001, 2018.
[39] Z. Wang and S. Hirai, "A 3D printed soft gripper integrated with curvature sensor for studying soft grasping," IEEE/SICE Int. Symp. Sys. Integ., 2016, pp. 629-633.

[40] K. Elgeneidy, N. Lohse and M. Jackson, "Bending angle prediction and control of soft pneumatic actuators with embedded flex sensors - A data-driven approach," Mechatron., vol. 50, pp. 234-247, 2018.

[41] Y. Chen, S. Guo, C. Li, H. Yang and L. Hao, "Size recognition and adaptive grasping using an integration of actuating and sensing soft pneumatic gripper," Robot. Auton. Syst., vol. 104, pp. 14-24, 2018.

[42] B.S. Homberg, R.K. Katzschmann, M.R. Dogar and D. Rus, "Haptic identification of objects using a modular soft robotic gripper," in Proc. IEEE/RSJ Int. Conf. Intell. Robots Syst., 2015, pp. 1698-1705.

[43] B. Shih, D. Drotman, C. Christianson, Z. Huo, R. White, H.I. Christensen and M.T. Tolley, "Custom soft robotic gripper sensor skins for haptic object visualization," in Proc. IEEE/RSJ Int. Conf. Intell. Robots Syst., 2017, pp. 494-501.

[44] B.S. Homberg, R.K. Katzschmann, M.R. Dogar and D. Rus, "Robust proprioceptive grasping with a soft robot hand," Auton. Robots, pp. 116, 2018.

[45] J. Nassour, V. Ghadiya, V. Hugel and F.H. Hamker, "Design of new Sensory Soft Hand: Combining air-pump actuation with superimposed curvature and pressure sensors," in Proc. IEEE Int. Conf. Soft Robot., 2018, pp. 164-169.

[46] A.J. Spiers, M.V. Liarokapis, B. Calli and A.M. Dollar, "Single-grasp object classification and feature extraction with simple robot hands and tactile sensors," IEEE Trans. Haptics, vol. 9, no. 2, pp. 207-220, 2016.

[47] J. Gandarias, J. Gómez-de-Gabriel and A. García-Cerezo, "Enhancing Perception with Tactile Object Recognition in Adaptive Grippers for Human-Robot Interaction," Sens., vol. 18, no. 3, pp. 692, 2018.

[48] A. Schneider, J. Sturm, C. Stachniss, M. Reisert, H. Burkhardt and W. Burgard, "Object identification with tactile sensors using bag-offeatures," in Proc. IEEE/RSJ Int. Conf. Intell. Robots Syst., 2009, pp. 243-248.

[49] U. Martinez-Hernandez, T.J. Dodd and T.J. Prescott, "Feeling the shape: active exploration behaviors for object recognition with a robotic hand," IEEE Trans. Syst., Man, and Cybern. Syst., vol PP, no. 99, pp. 1-10, 2017.

[50] J.A. Fishel and G.E. Loeb, "Bayesian exploration for intelligent identification of textures," Frontiers in Neurorob., vol. 6, pp. 1-20, 2012.

[51] P. Hebert, T. Howard, N. Hudson, J. Ma and J.W. Burdick, "The next best touch for model-based localization," in Proc. IEEE Int. Conf. Robot. Autom., 2013, pp. 99-106.

[52] L. Bykerk, D. Liu and K. Waldron, "A topology optimisation based design of a compliant gripper for grasping objects with irregular shapes," in Proc. IEEE/ASME Int. Conf. Adv. Intell. Mechatron., 2016, pp. 383388.

[53] M.R. Cutkosky, "On grasp choice, grasp models, and the design of hands for manufacturing tasks," IEEE Trans. Robot. Automat., vol. 5, no. 3, 1989.

Lili Bykerk received the B.Eng. degree in mechanical and mechatronic engineering from the University of Technology Sydney (UTS), Ultimo, NSW, Australia in 2014. She is currently pursuing the Ph.D. degree in engineering (robotics) with the Centre for Autonomous Systems, UTS. Her current research interests include soft robotics and touch-based object identification methods for applications in truss structure environments.

Phillip Quin received the B.Sc. degree in computer science, majoring in robotics from the University of New South Wales (UNSW). In 2016 he received his Ph.D. in engineering (robotics) at the University of Technology Sydney (UTS), Ultimo, NSW, Australia.

Dikai Liu (M06) is a Professor of mechanical and mechatronic engineering with the Centre for Autonomous Systems, University of Technology Sydney (UTS), Ultimo, NSW, Australia. His current research interest is robotics, including perception, mapping, motion planning, physical human-robot interaction, multirobot coordination and control. 